\title{
Matroid inequalities from electrical network theory
}

\author{
David G. Wagner* \\ Department of Combinatorics and Optimization \\ University of Waterloo, Waterloo, Ontario, Canada. \\ dgwagner@math. uwaterloo.ca
}

Submitted: Jul 7, 2004; Accepted: Dec 16, 2004; Published: Apr 13, 2005

Mathematics Subject Classifications: 05B35; 05A20, 05A15.

\begin{abstract}
In 1981, Stanley applied the Aleksandrov-Fenchel Inequalities to prove a logarithmic concavity theorem for regular matroids. Using ideas from electrical network theory we prove a generalization of this for the wider class of matroids with the "half-plane property". Then we explore a nest of inequalities for weighted basisgenerating polynomials that are related to these ideas. As a first result from this investigation we find that every matroid of rank three or corank three satisfies a condition only slightly weaker than the conclusion of Stanley's theorem.
\end{abstract}

\section{Dedicated with great admiration \\ to Richard Stanley \\ on the occasion of his 60th birthday.}

\section{Introduction.}

In 1981, Stanley [15] applied the Aleksandrov-Fenchel Inequalities to prove the following logarithmic concavity result:

Theorem 1.1 (Stanley, 1981) Let $\mathcal{M}$ be a matroid, and let $\pi=\left(S, T, C_{1}, \ldots, C_{k}\right)$ be an ordered partition of the ground-set $E$ of $\mathcal{M}$ into pairwise disjoint nonempty subsets, and fix nonnegative integers $c_{1}, \ldots, c_{k}$. For each $0 \leq j \leq|S|$, let $M_{j}(\pi)$ be the number of bases $B$ of $\mathcal{M}$ such that $|B \cap S|=j$ and $\left|B \cap C_{i}\right|=c_{i}$ for all $1 \leq i \leq k$. If $\mathcal{M}$ is regular then for each $1 \leq j \leq|S|-1$,

$$
\frac{M_{j}(\pi)^{2}}{\left(\begin{array}{c}
|S| \\
j
\end{array}\right)^{2}} \geq \frac{M_{j-1}(\pi)}{\left(\begin{array}{c}
|S| \\
j-1
\end{array}\right)} \cdot \frac{M_{j+1}(\pi)}{\left(\begin{array}{c}
|S| \\
j+1
\end{array}\right)} .
$$

*Research supported by the Natural Sciences and Engineering Research Council of Canada under operating grant OGP0105392. 
Stanley's proof proceeds by constructing a set of zonotopes with the desired mixed volumes.

A few years later, answering a question raised by Stanley, Godsil [7] strengthened this conclusion as follows:

Theorem 1.2 (Godsil, 1984) With the hypotheses and notation of Theorem 1.1, the polynomial $\sum_{j=0}^{|S|} M_{j}(\pi) x^{j}$ has only real (nonpositive) zeros.

The well-known Newton's Inequalities (item (51) of [8]) show that Theorem 1.2 implies Theorem 1.1. Godsil's proof employs a determinantal theorem used by Schneider [13] to prove the Aleksandrov-Fenchel Inequalities.

The aim of this paper is to publicize a recent extension of these results (Theorem 4.5 of [4]), to collect the scattered details of its proof into a self-contained whole, and to present some preliminary findings on related inequalities. It can also be regarded as an idiosyncratic introduction to the literature on the half-plane property, Rayleigh monotonicity, and related concepts $[1,2,3,4,5,6,10,12,14,16]$. We extend Theorem 1.2 in two directions - by relaxing the hypothesis and by strengthening the conclusion. For the hypothesis, we replace the condition that the matroid is regular by the weaker condition that the matroid has the half-plane property explained in Section 2. We strengthen the conclusion by introducing positive real weights $\mathbf{y}:=\left\{y_{e}: e \in E\right\}$ on the elements of the ground-set $E(\mathcal{M})$. The weight of a basis $B$ of $\mathcal{M}$ is then $\mathbf{y}^{B}:=\prod_{e \in B} y_{e}$, and with the notation of Theorem 1.1 we let $M_{j}(\pi, \mathbf{y}):=\sum_{B} \mathbf{y}^{B}$ with the sum over all bases $B$ of $\mathcal{M}$ such that $|B \cap S|=j$ and $\left|B \cap C_{i}\right|=c_{i}$ for all $1 \leq i \leq k$.

Theorem 1.3 (Theorem 4.5 of [4]) With the above hypotheses and notation, if $\mathcal{M}$ has the half-plane property then the polynomial

$$
\sum_{j=0}^{|S|} M_{j}(\pi, \mathbf{y}) x^{j}
$$

has only real (nonpositive) zeros.

\section{Electrical Networks and Matroids.}

Our proof of Theorem 1.3 builds on ideas originating with Kirchhoff's formula for the effective conductance of a (linear, resistive, direct current) electrical network. Such a network is represented by a graph $G=(V, E)$ with real positive weights $\mathbf{y}:=\left\{y_{e}: e \in E\right\}$ specifying the conductance of each edge of the graph. We use the notation

$$
G(\mathbf{y}):=\sum_{T} \mathbf{y}^{T}
$$

for the sum of $\mathbf{y}^{T}:=\prod_{e \in T} y_{e}$ over all spanning trees $T$ of $G$, and $\mathbf{y}>\mathbf{0}$ to indicate that every edge-weight is positive. Fixing two vertices $v, w \in V$, the effective conductance of 
the network measured between $v$ and $w$ is - by Kirchhoff's Formula [9] -

$$
y_{v w}(G ; \mathbf{y})=\frac{H^{f}(\mathbf{y})}{H_{f}(\mathbf{y})}
$$

Here $H$ denotes the graph obtained from $G$ by adjoining a new edge $f$ with ends $v$ and $w, H^{f}=G$ is $H$ with $f$ deleted, and $H_{f}$ is $H$ with $f$ contracted.

One intuitive property of electrical networks is that if the conductance $y_{e}$ of some edge $e$ of $G$ is increased, then $y_{v w}(G ; \mathbf{y})$ does not decrease. That is,

$$
\frac{\partial}{\partial y_{e}} y_{v w}(G ; \mathbf{y}) \geq 0
$$

This property is known as Rayleigh monotonicity. After some calculation using $H_{f}(\mathbf{y})=$ $H_{f}^{e}(\mathbf{y})+y_{e} H_{e f}(\mathbf{y})$ et cetera, this is found to be equivalent to the condition that if $\mathbf{y}>\mathbf{0}$ then

$$
H_{e}^{f}(\mathbf{y}) H_{f}^{e}(\mathbf{y}) \geq H_{e f}(\mathbf{y}) H^{e f}(\mathbf{y}) .
$$

(The deletion/contraction notation is extended in the obvious way.)

A less obvious property of the effective conductance is that if every $y_{e}$ is a complex number with positive real part then $y_{v w}(G ; \mathbf{y})$ is a complex number with nonnegative real part. Physically, this corresponds to the fact that if every edge of an alternating current circuit dissipates energy then the whole network cannot produce energy. Some minor hijinx with the equation $H(\mathbf{y})=H^{f}(\mathbf{y})+y_{f} H_{f}(\mathbf{y})$ shows that this is equivalent to the condition that if $\operatorname{Re}\left(y_{e}\right)>0$ for all $e \in E(H)$ then $H(\mathbf{y}) \neq 0$. Such a polynomial $H(\mathbf{y})$ is said to have the half-plane property.

The combinatorics of the preceeding three paragraphs carries over mutatis mutandis to matroids in general. In place of a graph $G$ we have a matroid $\mathcal{M}$. The edge-weights $\mathbf{y}$ become weights on the ground-set $E(\mathcal{M})$ of $\mathcal{M}$. In place of the spanning tree generating function $G(\mathbf{y})$ we have the basis generating function

$$
M(\mathbf{y}):=\sum_{B \in \mathcal{M}} \mathbf{y}^{B}
$$

Since this is insensitive to loops we might as well think of $\mathcal{M}$ as given by its set of bases. For disjoint subsets $I, J \subseteq E$ the contraction of $I$ and deletion of $J$ from $\mathcal{M}$ is given by

$$
\mathcal{M}_{I}^{J}:=\{B \backslash I: B \in \mathcal{M} \text { and } I \subseteq B \subseteq E \backslash J\}
$$

Rayleigh monotonicity corresponds to the inequalities

$$
M_{e}^{f}(\mathbf{y}) M_{f}^{e}(\mathbf{y}) \geq M_{e f}(\mathbf{y}) M^{e f}(\mathbf{y})
$$

for all $e, f \in E$ and $\mathbf{y}>\mathbf{0}$. A matroid satisfying these inequalities is called a Rayleigh matroid. If the basis generating polynomial $M(\mathbf{y})$ has the half-plane property then we say that $\mathcal{M}$ has the half-plane property, or is a HPP matroid. 


\section{The Half-Plane Property.}

Our first item of business is to show that every regular matroid is a HPP matroid. For graphs, Proposition 3.1 is part of the "folklore" of electrical engineering. We take it from Corollary 8.2(a) and Theorem 8.9 of [3], but include the short and interesting proof for completeness.

A matrix $A$ of complex numbers is a sixth-root of unity matrix provided that every nonzero minor of $A$ is a sixth-root of unity. A matroid $\mathcal{M}$ is a sixth-root of unity matroid provided that it can be represented over the complex numbers by a sixth-root of unity matrix. For example, every regular matroid is a sixth-root of unity matroid. Whittle [17] has shown that a matroid is a sixth-root of unity matroid if and only if it is representable over both $G F(3)$ and $G F(4)$. (It is worth noting that Godsil's proof can be adapted to prove Theorem 1.3 in the special case of sixth-root of unity matroids.)

Proposition 3.1 Every sixth-root of unity matroid is a HPP matroid.

Proof. Let $A$ be a sixth-root of unity matrix of full row-rank $r$, representing the matroid $\mathcal{M}$, and let $A^{*}$ denote the conjugate transpose of $A$. Index the columns of $A$ by the set $E$, and let $Y:=\operatorname{diag}\left(y_{e}: e \in E\right)$ be a diagonal matrix of indeterminates. For an $r$-element subset $S \subseteq E$, let $A[S]$ denote the square submatrix of $A$ supported on the set $S$ of columns. Since $A$ is a sixth-root of unity matrix, either $\operatorname{det} A[S]=0$ or $|\operatorname{det} A[S]|=1$. Thus, by the Binet-Cauchy formula,

$$
\operatorname{det}\left(A Y A^{*}\right)=\sum_{S \subseteq E:|S|=r}|\operatorname{det} A[S]|^{2} \mathbf{y}^{S}=M(\mathbf{y})
$$

is the basis-generating polynomial of $\mathcal{M}$.

Now we claim that if $\operatorname{Re}\left(y_{e}\right)>0$ for all $e \in E$, then $A Y A^{*}$ is nonsingular. This suffices to prove the result. Consider any nonzero vector $\mathbf{v} \in \mathbb{C}^{r}$. Then $A^{*} \mathbf{v} \neq \mathbf{0}$ since the columns of $A^{*}$ are linearly independent. Therefore

$$
\mathbf{v}^{*} A Y A^{*} \mathbf{v}=\sum_{e \in E} y_{e}\left|\left(A^{*} \mathbf{v}\right)_{e}\right|^{2}
$$

has strictly positive real part, since for all $e \in E$ the numbers $\left|\left(A^{*} \mathbf{v}\right)_{e}\right|^{2}$ are nonnegative reals and at least one of these is positive. In particular, for any nonzero $\mathbf{v} \in \mathbb{C}^{r}$, the vector $A Y A^{*} \mathbf{v}$ is nonzero. It follows that $A Y A^{*}$ is nonsingular, completing the proof.

The same proof shows that for any complex matrix $A$ of full row-rank $r$, the polynomial

$$
\operatorname{det}\left(A Y A^{*}\right)=\sum_{S \subseteq E:|S|=r}|\operatorname{det} A[S]|^{2} \mathbf{y}^{S}
$$

has the half-plane property. The weighted analogue of Rayleigh monotonicity in this case is discussed from a probabilistic point of view by Lyons [10]. It is a surprising fact that a complex matrix $A$ of full row-rank $r$ has $|\operatorname{det} A[S]|^{2}=1$ for all nonzero rank $r$ minors if and only if $A$ represents a sixth-root of unity matroid (Theorem 8.9 of [3]).

The proof of Theorem 1.3 is based on the following lemmas regarding the half-plane property. The paper [3] gives a much more thorough development of the theory. 
Lemma 3.2 Let $P(\mathbf{y})$ be a polynomial in the variables $\mathbf{y}=\left\{y_{e}: e \in E\right\}$, let $e \in E$, and let the degree of $y_{e}$ in $P$ be $n$. If $P(\mathbf{y})$ has the half-plane property then $y_{e}^{n} P\left(\left\{y_{f}: f \neq\right.\right.$ e\}, $1 / y_{e}$ ) has the half-plane property.

Proof. This follows immediately from the fact that $\operatorname{Re}\left(1 / y_{e}\right)>0$ if and only if $\operatorname{Re}\left(y_{e}\right)>0$.

Lemma 3.3 ([3], Proposition 2.8) Let $P(\mathbf{y})$ be a polynomial in the variables $\mathbf{y}=\left\{y_{e}\right.$ : $e \in E\}$. For any $e \in E$, if $P$ has the half-plane property then $\partial P / \partial y_{e}$ has the half-plane property.

Proof. Fix complex values with positive real parts for every $y_{f}$ with $f \in E \backslash\{e\}$. The result of these substitutions is a univariate polynomial $F\left(y_{e}\right)$ all the roots of which have nonpositive real part. Thus

$$
F\left(y_{e}\right)=C \prod_{j=1}^{n}\left(y_{e}+\theta_{j}\right)
$$

with $\operatorname{Re}\left(\theta_{j}\right) \geq 0$ for all $1 \leq i \leq n$. It follows that if $\operatorname{Re}\left(y_{e}\right)>0$ then the real part of

$$
\frac{F^{\prime}\left(y_{e}\right)}{F\left(y_{e}\right)}=\sum_{j=1}^{n} \frac{1}{y_{e}+\theta_{j}}
$$

is also strictly positive. In particular $F^{\prime}\left(y_{e}\right) \neq 0$. It follows that $\partial P / \partial y_{e}$ has the half-plane property.

Corollary 3.4 ([5], Theorem 18, or [3], Proposition 3.4.) Let $P(\mathbf{y})$ be a polynomial in the variables $\mathbf{y}=\left\{y_{e}: e \in E\right\}$, fix $e \in E$, and let $P(\mathbf{y})=\sum_{j=0}^{n} P_{j}\left(\left\{y_{f}: f \neq e\right\}\right) y_{e}^{j}$. If $P$ has the half-plane property then each $P_{j}$ has the half-plane property.

Proof. Let $n$ be the degree of $P(\mathbf{y})$ in the variable $y_{e}$. Let $A:=\partial^{j} P / \partial y_{e}^{j}, B:=y_{e}^{n-j} A\left(\left\{y_{f}\right.\right.$ : $\left.f \neq e\}, 1 / y_{e}\right)$, and $C:=\partial^{n-j-1} B / \partial y_{e}^{n-j-1}$. Then $C(\mathbf{y})$ is a nonzero multiple of $P_{j}$, and has the half-plane property by Lemmas 3.2 and 3.3.

Lemma 3.5 ([3], Proposition 5.2) Let $P(\mathbf{y})$ be a homogeneous polynomial in the variables $\mathbf{y}=\left\{y_{e}: e \in E\right\}$. For sets of nonnegative real numbers $\mathbf{a}=\left\{a_{e}: e \in E\right\}$ and $\mathbf{b}=\left\{b_{e}: e \in E\right\}$, let $P(\mathbf{a} x+\mathbf{b})$ be the polyomial obtained by substituting $y_{e}=a_{e} x+b_{e}$ for each $e \in E$. The following are equivalent:

(a) $P(\mathbf{y})$ has the half-plane property;

(b) for all sets of nonnegative real numbers $\mathbf{a}$ and $\mathbf{b}, P(\mathbf{a} x+\mathbf{b})$ has only real (nonpositive) zeros.

Proof. To see that (a) implies (b), suppose that $\xi$ is a zero of $P(\mathbf{a} x+\mathbf{b})$ that is not a nonpositive real. Then there are complex numbers $z$ and $w$ with positive real part such that $z / w=\xi$. If $P(\mathbf{y})$ is homogeneous of degree $r$ then $P(\mathbf{a} z+\mathbf{b} w)=w^{r} P(\mathbf{a} \xi+\mathbf{b})=0$, showing that $P(\mathbf{y})$ fails to have the half-plane property. 
To see that (b) implies (a), consider any set of values $\left\{y_{e}: e \in E\right\}$ with $\operatorname{Re}\left(y_{e}\right)>0$ for all $e \in E$. There are complex numbers $z$ and $w$ with positive real parts such that all the $y_{e}$ are in the convex cone generated by $z$ and $w$. That is, for each $e \in E$ there are nonnegative reals $a_{e}$ and $b_{e}$ such that $y_{e}=a_{e} z+b_{e} w$. Now $P(\mathbf{y})=w^{r} P(\mathbf{a} \xi+\mathbf{b})$ in which $\xi=z / w$ is not in the interval $(-\infty, 0]$, and so $P(\mathbf{y}) \neq 0$.

We can now prove Theorem 1.3.

Proof of Theorem 1.3. Let $\mathcal{M}$ be a HPP matroid and fix $\mathbf{y}>\mathbf{0}$. Let $s, t$, and $z_{1}, \ldots, z_{k}$ be indeterminates, and for $e \in E$ put

$$
u_{e}:= \begin{cases}y_{e} s & \text { if } \quad e \in S, \\ y_{e} t & \text { if } e \in T \\ y_{e} z_{i} & \text { if } \quad e \in C_{i}\end{cases}
$$

Then $M(\mathbf{u})$ is a homogeneous polynomial with the half-plane property in the variables $s, t, z_{1}, \ldots, z_{k}$. By repeated application of Corollary 3.4 , the coefficient $M_{\mathbf{c}}(s, t)$ of $z_{1}^{c_{1}} \cdots z_{k}^{c_{k}}$ in $M(\mathbf{u})$ also has the half-plane property, and is homogeneous. In fact,

$$
M_{\mathbf{c}}(s, t)=\sum_{j=0}^{|S|} M_{j}(\pi, \mathbf{y}) s^{j} t^{d-j}
$$

in which $d=\operatorname{rank}(\mathcal{M})-\left(c_{1}+\cdots+c_{k}\right)$. Upon substituting $s=x$ and $t=1$ in $M_{\mathbf{c}}(s, t)$, Lemma 3.5 implies that $\sum_{j=0}^{|S|} M_{j}(\pi, \mathbf{y}) x^{j}$ has only real (nonpositive) zeros, as claimed.

\section{Between HPP and Rayleigh.}

This updated version of Stanley's theorem provides a link between the half-plane property and Rayleigh monotonicity in the context of matroids. Only the $k=0$ case of the theorem is needed. (In fact the $k=0$ case is equivalent to the general case by various properties of HPP matroids.) For a subset $S \subseteq E(\mathcal{M})$ and natural number $j$, let $M_{j}(S, \mathbf{y}):=\sum_{B} \mathbf{y}^{B}$, with the sum over all bases $B$ of $\mathcal{M}$ such that $|B \cap S|=j$. For each positive integer $m$, consider the following conditions on a matroid $\mathcal{M}$ :

$\mathbf{R Z}[m]$ : If $\mathbf{y}>\mathbf{0}$ then for all $S \subseteq E$ with $|S| \leq m$ the polynomial $\sum_{j=0}^{|S|} M_{j}(S, \mathbf{y}) x^{j}$ has only real zeros.

BLC $[m]$ : If $\mathbf{y}>\mathbf{0}$ then for all $S \subseteq E$ with $|S|=n \leq m$ and all $1 \leq j \leq n-1$,

$$
M_{j}(S, \mathbf{y})^{2} \geq\left[1+\frac{n+1}{j(n-j)}\right] M_{j-1}(S, \mathbf{y}) M_{j+1}(S, \mathbf{y})
$$

The mnemonics are for "real zeros" and "binomial logarithmic concavity", respectively. We also say that a matroid satisfies RZ if it satisfies RZ $[m]$ for all $m$, and that it satisfies 
BLC if it satisfies $\mathrm{BLC}[m]$ for all $m$. Our BLC is a weighted strengthening of Stanley's "Property P".

The $k=0$ case of Theorem 1.3 implies that a HPP matroid satisfies RZ, and Newton's Inequalities show that RZ $[m]$ implies $\mathrm{BLC}[m]$ for every $m$. The implications $\mathrm{RZ}[m] \Longrightarrow$ $\mathrm{RZ}[m-1]$ and $\mathrm{BLC}[m] \Longrightarrow \mathrm{BLC}[m-1]$ are trivial, as are the conditions RZ[1] and $\mathrm{BLC}[1]$. Thus, the weakest nontrivial condition among these is BLC[2]. This is in fact equivalent to Rayleigh monotonicity, as the remarks after Theorem 4.3 show.

Proposition 4.1 ([4], Corollary 4.9) Every HPP matroid is a Rayleigh matroid.

Proof. Theorem 1.3 shows that every HPP matroid satisfies BLC[2]. We show here that if $\mathcal{M}$ satisfies BLC[2] then it is Rayleigh. So, let $\mathbf{y}>\mathbf{0}$ be positive weights on $E(\mathcal{M})$ and let $S=\{e, f\} \subseteq E$. To prove the Rayleigh inequality $M_{e}^{f}(\mathbf{y}) M_{f}^{e}(\mathbf{y}) \geq M_{e f}(\mathbf{y}) M^{e f}(\mathbf{y})$ it suffices to consider the case in which both $e$ and $f$ are neither loops nor coloops. In this case, define another set of weights by $w_{e}:=M_{f}^{e}(\mathbf{y})$ and $w_{f}:=M_{e}^{f}(\mathbf{y})$ and $w_{g}:=y_{g}$ for all $g \in E \backslash\{e, f\}$. Then, since $\mathbf{w}>0$ and $\mathcal{M}$ satisfies BLC[2], the inequality

$$
M_{1}(S, \mathbf{w})^{2} \geq 4 M_{0}(S, \mathbf{w}) M_{2}(S, \mathbf{w})
$$

holds. This can be expanded to

$$
\left(w_{e} M_{e}^{f}(\mathbf{w})+w_{f} M_{f}^{e}(\mathbf{w})\right)^{2} \geq 4 M^{e f}(\mathbf{w}) w_{e} w_{f} M_{e f}(\mathbf{w}),
$$

and finally to

$$
4\left(M_{e}^{f}(\mathbf{y}) M_{f}^{e}(\mathbf{y})\right)^{2} \geq 4 M_{e f}(\mathbf{y}) M^{e f}(\mathbf{y}) M_{e}^{f}(\mathbf{y}) M_{f}^{e}(\mathbf{y}) .
$$

Cancellation of common (positive) factors from both sides yields the desired inequality.

In view of the implication $\mathrm{BLC}[2] \Longrightarrow$ Rayleigh, it is interesting to look for conditions (other than HPP) which imply BLC $[m]$ for various $m$. This is further motivated by Stanley's application of "Property P" to Mason's Conjecture - see Theorem 2.9 of [15]. The following hierarchies of strict root-binomial logarithmic concavity and strict logarithmic concavity conditions are also interesting:

$\sqrt{\mathbf{B L C}}[m]$ If $\mathbf{y}>\mathbf{0}$ then for all $S \subseteq E$ with $|S|=n \leq m$ and all $1 \leq j \leq n-1$, if $M_{j}(S, \mathbf{y}) \neq 0$ then

$$
M_{j}(S, \mathbf{y})^{2}>\left[1+\frac{1}{\min (j, n-j)}\right] M_{j-1}(S, \mathbf{y}) M_{j+1}(S, \mathbf{y}) .
$$

$\mathbf{S L C}[m]:$ If $\mathbf{y}>\mathbf{0}$ then for all $S \subseteq E$ with $|S| \leq m$ and all $1 \leq j \leq|S|-1$, if $M_{j}(S, \mathbf{y}) \neq 0$ then

$$
M_{j}(S, \mathbf{y})^{2}>M_{j-1}(S, \mathbf{y}) M_{j+1}(S, \mathbf{y}) .
$$


We also say that a matroid satisfies $\sqrt{\mathrm{BLC}}$ if it satisfies $\sqrt{\mathrm{BLC}}[m]$ for all $m$, and satisfies SLC if it satisfies $\mathrm{SLC}[m]$ for all $m$. The inequalities

$$
1+\frac{1}{\min (j, n-j)}<1+\frac{n+1}{j(n-j)} \leq\left[1+\frac{1}{\min (j, n-j)}\right]^{2}
$$

for $1 \leq j \leq n-1$ show that $\mathrm{BLC}[m]$ implies $\sqrt{\mathrm{BLC}}[m]$ for every $m$, and motivate this somewhat odd terminology. Clearly $\sqrt{\mathrm{BLC}}[m]$ implies SLC $[m]$ for every $m$.

In the following calculations we will usually omit explicit reference to the variables $\mathbf{y}$ unless a particular substitution must be emphasized. For $\mathcal{M}$ a matroid, $S \subseteq E(\mathcal{M})$, and $k$ a natural number, let

$$
\Psi_{k} M S:=\sum_{A \subseteq S:|A|=k} M_{A}^{S \backslash A} M_{S \backslash A}^{A}
$$

For example,

$$
\Psi_{2} M\{a, b, c\}:=M_{a b}^{c} M_{c}^{a b}+M_{a c}^{b} M_{b}^{a c}+M_{b c}^{a} M_{a}^{b c} .
$$

Notice that in general if $|S|=n$ then $\Psi_{k} M S=\Psi_{n-k} M S$. The Rayleigh inequality is that

$$
\Psi_{1} M\{e, f\} \geq 2 \Psi_{2} M\{e, f\} .
$$

This suggests several possible generalizations, among which we will concentrate here on the following. For each integer $k \geq 1$ and real $\lambda>0$, say that $\mathcal{M}$ is $k$-th level Rayleigh of strength $\lambda$ provided that:

$\lambda$-Ray $[k]:$ If $\mathbf{y}>\mathbf{0}$ then for every $S \subseteq E(\mathcal{M})$ with $|S|=2 k$,

$$
\Psi_{k} M S \geq \lambda \Psi_{k+1} M S
$$

The condition 2-Ray[1] is exactly the Rayleigh condition. In general, each term on the LHS of $\lambda$-Ray $[k]$ occurs twice. Proposition 4.6 below shows that $(1+1 / k)$-Ray $[k]$ is an especially natural strength for these conditions. Interestingly, this lies right between two of the most useful strengths for these conditions. As an example, the inequality for $(3 / 2)$-Ray[2] is

$$
\begin{aligned}
& 4\left[M_{a b}^{c d} M_{c d}^{a b}+M_{a c}^{b d} M_{b d}^{a c}+M_{a d}^{b c} M_{b c}^{a d}\right] \\
\geq & 3\left[M_{b c d}^{a} M_{a}^{b c d}+M_{a c d}^{b} M_{b}^{a c d}+M_{a b d}^{c} M_{c}^{a b d}+M_{a b c}^{d} M_{d}^{a b c}\right] .
\end{aligned}
$$

Lemma 4.2 (a) For each $k \geq 1$ and $\lambda>0$, the class of matroids satisfying $\lambda$-Ray $[k]$ is closed by taking duals and minors.

(b) For each $m \geq 1$, the class of matroids satisfying $\mathrm{BLC}[m]$ is closed by taking duals and minors.

(c) For each $m \geq 1$, the class of matroids satisfying $\sqrt{\mathrm{BLC}}[m]$ is closed by taking duals and minors.

(d) For each $m \geq 1$, the class of matroids satisfying $\mathrm{SLC}[m]$ is closed by taking duals and minors. 
Sketch of proof. For the matroid $\mathcal{M}^{*}$ dual to $\mathcal{M}$ we have $M^{*}(\mathbf{y})=\mathbf{y}^{E} M(\mathbf{1} / \mathbf{y})$. From this it follows that for $0 \leq k \leq n=|S|$,

$$
\Psi_{k} M^{*} S(\mathbf{y})=\left(\mathbf{y}^{E \backslash S}\right)^{2} \Psi_{n-k} M S(\mathbf{1} / \mathbf{y}) .
$$

Since $\mathbf{y}>\mathbf{0}$ is arbitrary, one sees that $\lambda$-Ray $[k]$ for $\mathcal{M}$ implies $\lambda$-Ray $[k]$ for $\mathcal{M}^{*}$. Similarly

$$
M_{j}(S, \mathbf{y})^{2}>M_{j-1}(S, \mathbf{y}) M_{j+1}(S, \mathbf{y})
$$

implies

$$
\left(\mathbf{y}^{E}\right)^{2} M_{n-j}^{*}(S, \mathbf{1} / \mathbf{y})^{2}>\left(\mathbf{y}^{E}\right)^{2} M_{n-j+1}^{*}(S, \mathbf{1} / \mathbf{y}) M_{n-j-1}^{*}(S, \mathbf{1} / \mathbf{y}) .
$$

From this it follows that SLC $[m]$ for $\mathcal{M}$ implies SLC $[m]$ for $\mathcal{M}^{*}$. Analogous arguments work for BLC $[m]$ and $\sqrt{\mathrm{BLC}}[m]$.

For a set $S \subseteq E(\mathcal{M})$ and $g \in E \backslash S$, we have

$$
\Psi_{k} M S(\mathbf{y})=y_{g}^{2} \Psi_{k} M_{g} S(\mathbf{y})+y_{g} Q(\mathbf{y})+\Psi_{k} M^{g} S(\mathbf{y})
$$

for some polynomial $Q(\mathbf{y})$. If $\mathcal{M}$ satisfies $\lambda$-Ray $[k]$ then taking the limit as $y_{g} \rightarrow 0$ shows that the deletion $\mathcal{M}^{g}$ satisfies $\lambda$-Ray $[k]$. Similarly, multiplying by $1 / y_{g}^{2}$ and taking the limit as $y_{g} \rightarrow \infty$ shows that the contraction $\mathcal{M}_{g}$ satisfies $\lambda$-Ray $[k]$. The case of a general minor is obtained by iteration of these two cases. Analogous arguments work for BLC $[m]$, $\sqrt{\mathrm{BLC}}[m]$, and $\mathrm{SLC}[m]$.

Theorem 4.3 If $\mathcal{M}$ satisfies 2 -Ray[1] and $(1+1 / k)^{2}$-Ray $[k]$ for all $2 \leq k \leq m$ then $\mathcal{M}$ satisfies $\mathrm{BLC}[2 m+1]$.

Proof. The proof uses the following elementary inequality: for $N \geq 2$ real numbers $R_{1}, \ldots, R_{N}$

$$
\left(R_{1}+\cdots+R_{N}\right)^{2} \geq \frac{2 N}{N-1} \sum_{\{i, j\} \subseteq\{1, \ldots, N\}} R_{i} R_{j} .
$$

Assume that $\mathcal{M}$ satisfies the hypothesis, and fix positive real weights $\mathbf{y}>\mathbf{0}$. To show that $\mathcal{M}$ satisfies BLC $[2 m+1]$, consider a subset $S \subseteq E$ with $|S|=n \leq 2 m+1$ and an index $1 \leq j \leq n-1$. We must show that

$$
M_{j}(S)^{2} \geq\left[1+\frac{n+1}{j(n-j)}\right] M_{j-1}(S) M_{j+1}(S) .
$$

From the definition we have

$$
M_{j}(S)=\sum_{A \subset S:|A|=j} \mathbf{y}^{A} M_{A}^{S \backslash A} .
$$

The inequality (1) implies that

$$
M_{j}(S)^{2} \geq \frac{2 N}{N-1} \sum_{\{A, B\}} \mathbf{y}^{A} \mathbf{y}^{B} M_{A}^{S \backslash A} M_{B}^{S \backslash B},
$$


with $N:=\left(\begin{array}{l}n \\ j\end{array}\right)$ and the sum over all pairs of distinct $j$-element subsets of $S$. We collect terms on the right side according to the intersection $I:=A \cap B$ and union $U:=A \cup B$ of the indexing pair of sets $\{A, B\}$. With $i:=|I|$ and $k:=j-i$ we have $|U \backslash I|=2 k$ and the sum of the terms on the RHS of (2) with this fixed $I$ and $U$ is

$$
\begin{aligned}
& \frac{N}{N-1} \mathbf{y}^{I} \mathbf{y}^{U} \sum_{C \subset U \backslash I:|C|=k}\left(M_{I}^{S \backslash U}\right)_{C}^{U \backslash I \backslash C}\left(M_{I}^{S \backslash U}\right)_{U \backslash I \backslash C}^{C} \\
= & \frac{N}{N-1} \mathbf{y}^{I} \mathbf{y}^{U} \Psi_{k} M_{I}^{S \backslash U}(U \backslash I) .
\end{aligned}
$$

Therefore

$$
M_{j}(S)^{2} \geq \frac{N}{N-1} \sum_{k=1}^{h} \sum_{(I, U)} \mathbf{y}^{I} \mathbf{y}^{U} \Psi_{k} M_{I}^{S \backslash U}(U \backslash I)
$$

in which $h:=\min (j, n-j)$ and the inner sum is over all pairs of sets $I \subset U \subseteq S$ with $|U|+|I|=2 j$ and $|U \backslash I|=2 k$. Let $\lambda_{1}:=2$ and $\lambda_{k}:=(1+1 / k)^{2}$ for all $k \geq 2$. Since $k \leq h \leq m$ we are assuming that $\mathcal{M}$ satisfies $\lambda_{k}$-Ray $[k]$, and hence each minor $\mathcal{M}_{I}^{S \backslash U}$ satisfies $\lambda_{k}$-Ray $[k]$ by Lemma $4.2(\mathrm{a})$. It follows that

$$
M_{j}(S)^{2} \geq \frac{N}{N-1} \sum_{k=1}^{h} \lambda_{k} \sum_{(I, U)} \mathbf{y}^{I} \mathbf{y}^{U} \Psi_{k+1} M_{I}^{S \backslash U}(U \backslash I) .
$$

If $h \geq 3$ then for all $1 \leq k \leq h$,

$$
\lambda_{k} \geq \lambda_{h}=\left(1+\frac{1}{h}\right)^{2} \geq 1+\frac{n+1}{j(n-j)} .
$$

If $h=2$ and $n \geq 5$ then

$$
\lambda_{2}=\frac{9}{4}>2=\lambda_{1} \geq 1+\frac{n+1}{j(n-j)} .
$$

In these cases we conclude from (3) that

$$
\begin{aligned}
M_{j}(S)^{2} & \geq \frac{N}{N-1}\left[1+\frac{n+1}{j(n-j)}\right] \sum_{k=1}^{h} \sum_{(I, U)} \mathbf{y}^{I} \mathbf{y}^{U} \Psi_{k+1} M_{I}^{S \backslash U}(U \backslash I) \\
& =\frac{N}{N-1}\left[1+\frac{n+1}{j(n-j)}\right] M_{j-1}(S) M_{j+1}(S) .
\end{aligned}
$$

This implies the desired inequality in these cases.

If $h=1$ then either $j=1$ or $j=n-1$, so $k=1$ and $N=n$, and we conclude from (3) that

$$
\begin{aligned}
M_{j}(S)^{2} & \geq \frac{2 n}{n-1} \sum_{(I, U)} \mathbf{y}^{I} \mathbf{y}^{U} \Psi_{2} M_{I}^{S \backslash U}(U \backslash I) \\
& =\frac{2 n}{n-1} M_{j-1}(S) M_{j+1}(S) .
\end{aligned}
$$


This is the desired inequality when $j=1$ or $j=n-1$.

If $n \leq 3$ then $h=1$, so the only remaining case is $n=4$ and $h=2$. In this case $j=2$ and $N=6$, and since $\lambda_{2}=9 / 4>2=\lambda_{1}$, from (3) we conclude that

$$
\begin{aligned}
M_{2}(S)^{2} & \geq \frac{6}{5} \sum_{k=1}^{2} 2 \sum_{(I, U)} \mathbf{y}^{I} \mathbf{y}^{U} \Psi_{k+1} M_{I}^{S \backslash U}(U \backslash I) \\
& \geq \frac{9}{4} M_{1}(S) M_{3}(S) .
\end{aligned}
$$

This is the desired inequality in this case.

This completes the verification that $\mathcal{M}$ satisfies $\mathrm{BLC}[2 m+1]$.

Notice that Proposition 4.1 and the $m=1$ case of Theorem 4.3 show that the conditions BLC[3], BLC[2], and 2-Ray[1] are in fact equivalent - this is part of Theorem 4.8 of [4].

By examining the proof of Theorem 4.3 one sees the following.

Porism 4.4 (a) If $\mathcal{M}$ satisfies $(1+1 / k)$-Ray $[k]$ for all $1 \leq k \leq m$ then $\mathcal{M}$ satisfies $\sqrt{\mathrm{BLC}}[2 m+1]$.

(b) If $\mathcal{M}$ satisfies 1 -Ray $[k]$ for all $1 \leq k \leq m$ then $\mathcal{M}$ satisfies $\operatorname{SLC}[2 m+1]$.

Corollary 4.5 Let $\mathcal{M}$ be a matroid of rank $r$ with $|E|=\ell$ elements.

(a) If $\mathcal{M}$ satisfies 2 -Ray[1] and $(1+1 / k)^{2}$-Ray $[k]$ for all $2 \leq k \leq r-1$ then $\mathcal{M}$ satisfies BLC.

(b) If $\mathcal{M}$ satisfies $(1+1 / k)$-Ray $[k]$ for all $1 \leq k \leq r-1$ then $\mathcal{M}$ satisfies $\sqrt{\mathrm{BLC}}$.

(c) If $\mathcal{M}$ satisfies 1 -Ray $[k]$ for all $1 \leq k \leq \min (r-1,(\ell-2) / 4)$ then $\mathcal{M}$ satisfies SLC.

Proof. If $\mathcal{M}$ has rank $r$ then $\mathcal{M}$ satisfies any condition $\lambda$-Ray $[k]$ trivially for all $k \geq r$ because for any $S \subseteq E(\mathcal{M})$ with $|S|=2 k, \Psi_{k+1} M S=0$ identically. In part (a) Theorem 4.3 thus implies that $\mathcal{M}$ satisfies BLC $[2 m+1]$ for all $m$ - that is BLC. In part (b) Porism 4.4(a) supports the analogous implication. Similarly, in part (c) if $r-1 \leq(\ell-2) / 4$ then Porism 4.4(b) implies that $\mathcal{N}$ satisfies SLC. If $(\ell-2) / 4<r-1$ then Porism 4.4(b) implies that $\mathcal{M}$ satisfies $\operatorname{SLC}[\lfloor\ell / 2]]$. For any $S \subset E$ with $|S|=n \leq \ell / 2$ and any $1 \leq j \leq n$ we have $M_{j}(S)=M_{r-j}(E \backslash S)$. The SLC inequalities for the set $S$ thus imply the SLC inequalities for the set $E \backslash S$. It follows that $\mathcal{M}$ satisfies SLC.

One of course wants examples of matroids satisfying these higher level Rayleigh conditions. So far there are no very substantial results in this direction. Direct computations (with the aid of MAPLE) and ad hoc arguments have shown that the Kuratowski graphs $\mathrm{K}_{5}$ and $\mathrm{K}_{3,3}$ satisfy (3/2)-Ray[2] but not (9/4)-Ray[2]. The hypothesis of Theorem 4.3 thus seems to be extremely restrictive. The corresponding calculation for $\mathrm{K}_{6}$ is so far intractible. Similar computations for $\lambda$-Ray $[k]$ with $k \geq 3$ have not yet been tried.

One easy sufficient condition is the following naively optimistic generalization of the Rayleigh condition. 
Proposition 4.6 Assume that $\mathcal{M}$ is such that for all disjoint subsets $A$ and $B$ of $E(\mathcal{M})$ with $|A|=|B|=k$ and $b \in B$, if $\mathbf{y}>\mathbf{0}$ then

$$
\mathcal{M}_{A}^{B} M_{B}^{A} \geq M_{A b}^{B \backslash b} M_{B \backslash b}^{A b} .
$$

Then $\mathcal{M}$ satisfies $(1+1 / k)$-Ray $[k]$.

Proof. Let $\mathcal{M}$ be as in the hypothesis, let $\mathbf{y}>\mathbf{0}$, and fix a set $S \subseteq E(\mathcal{M})$ with $|S|=2 k$. To verify the inequality $k \Psi_{k} M S \geq(k+1) \Psi_{k+1} M S$, form a graph $G$ with bipartition $(X, Y)$ as follows. The vertices in $X$ are the $k$-element subsets of $S$ and the vertices of $Y$ are the $(k+1)$ element subsets of $S$. There is an edge from $A \in X$ to $A^{\prime} \in Y$ whenever $A \subset A^{\prime}$. Thus, every vertex of $X$ has degree $k$ and every vertex of $Y$ has degree $k+1$. To each edge $\{A, A b\}$ of $G$ associate the weight $M_{A}^{S \backslash A} M_{S \backslash A}^{A}$. The total weight assigned to the edges is thus

$$
k \sum_{A \in X} M_{A}^{S \backslash A} M_{S \backslash A}^{A}=k \Psi_{k} M S .
$$

On the other hand, for each $A^{\prime} \in Y$ the sum of the weights of edges incident with $A^{\prime}$ is

$$
\begin{aligned}
& \sum_{b \in A^{\prime}} M_{A^{\prime} \backslash b}^{S \backslash\left(A^{\prime} \backslash b\right)} M_{S \backslash\left(A^{\prime} \backslash b\right)}^{A^{\prime} \backslash b} \\
\geq & \sum_{b \in A^{\prime}} M_{A^{\prime}}^{S \backslash A^{\prime}} M_{S \backslash A^{\prime}}^{A^{\prime}}=(k+1) M_{A^{\prime}}^{S \backslash A^{\prime}} M_{S \backslash A^{\prime}}^{A^{\prime}} .
\end{aligned}
$$

It follows that the sum of the weights of the edges of $G$ is at least $(k+1) \Psi_{k+1} M S$, completing the proof.

Unfortunately, it is not hard to find planar graphs which fail to satisfy the hypothesis of Proposition 4.6 in the case $k=2$. An easy example is the 4 -wheel $\mathrm{W}_{4}$ formed by a cycle of four "rim" edges with the vertices joined to a new "hub" vertex by four "spoke" edges. The spoke edges are labelled $a, b, c, d$ in cyclic order, and the rim edges have weights $y$, $1, y, 1$ in cyclic order starting with the edge forming a triangle with $a$ and $b$. With $\mathcal{W}$ denoting the graphic matroid of $\mathrm{W}_{4}$, we have $W_{a b}^{c d}=W_{c d}^{a b}=2 y+1$ and $W_{a b c}^{d}=y+1$ and $W_{d}^{a b c}=2 y(y+1)$, so that the inequality

$$
W_{a b}^{c d} W_{c d}^{a b} \geq W_{a b c}^{d} W_{d}^{a b c}
$$

is equivalent to

$$
(2 y+1)^{2} \geq 2 y(y+1)^{2} .
$$

This inequality $2 y^{3} \leq 2 y+1$ is not satisfied for all $y>0$, so that $\mathcal{W}$ does not meet the hypothesis of Proposition 4.4. Note that $\mathcal{W}$ does satisfy (3/2)-Ray[2], however, since $\mathbf{W}_{4}$ is a minor of $\mathrm{K}_{5}$.

In general, the condition $(1+1 / k)$-Ray $[k]$ asserts that the inequalities in the hypothesis of Proposition 4.6 hold "locally on average" in some sense.

To close this section we show that the condition $\sqrt{\mathrm{BLC}}$ has consequences for Mason's Conjecture [11] - this is inspired by Theorem 2.9 of Stanley [15]. For a matroid $\mathcal{M}$ of 
rank $r$ and $0 \leq j \leq r$, let $I_{j}(\mathcal{M})$ denote the number of $j$-element independent sets of $\mathcal{M}$. Mason's Conjecture is that for any matroid, $I_{j}^{2} \geq I_{j-1} I_{j+1}$ for all $1 \leq j \leq r-1$. Let $\mathcal{M} \oplus \mathcal{U}_{\ell, \ell}$ denote the free extension of $\mathcal{M}$ by $\ell$ points, and let $T_{r}\left(\mathcal{M} \oplus \mathcal{U}_{\ell, \ell}\right)$ denote the truncation of this matroid down to rank $r$.

Theorem 4.7 Let $\mathcal{M}$ be a matroid of rank $r$. Assume that, for infinitely many integers $\ell \geq 1, T_{r}\left(\mathcal{M} \oplus \mathcal{U}_{\ell, \ell}\right)$ satisfies $\sqrt{\mathrm{BLC}}[\ell]$. Then $\mathcal{M}$ satisfies Mason's Conjecture.

Proof. Let $\mathcal{L}:=T_{r}\left(\mathcal{M} \oplus \mathcal{U}_{\ell, \ell}\right)$ for some $\ell \geq 1$, and let $S:=E(\mathcal{L}) \backslash E(\mathcal{M})$. For any $0 \leq j \leq r$ we have $L_{j}(S, \mathbf{1})=\left(\begin{array}{l}\ell \\ j\end{array}\right) I_{r-j}(\mathcal{M})$. If $1 \leq j \leq r-1$ and $\ell \geq 2 j$ is such that $\mathcal{L}$ satisfies $\sqrt{\mathrm{BLC}}[\ell]$ then

$$
L_{j}(S, \mathbf{1})^{2}>\left[1+\frac{1}{j}\right] L_{j-1}(S, \mathbf{1}) L_{j+1}(S, \mathbf{1}) .
$$

From this we obtain

$$
I_{r-j}(\mathcal{M})^{2}>\frac{\ell-j}{\ell-j+1} I_{r-j+1}(\mathcal{M}) I_{r-j-1}(\mathcal{M}) .
$$

Since this holds for a sequence of $\ell \rightarrow \infty$ we conclude that

$$
I_{r-j}(\mathcal{M})^{2} \geq I_{r-j+1}(\mathcal{M}) I_{r-j-1}(\mathcal{M}) .
$$

As this holds for all $1 \leq j \leq r-1, \mathcal{M}$ satisfies Mason's Conjecture.

\section{Matroids of rank three.}

In [16] it is shown that every matroid of rank (at most) three satisfies the Rayleigh condition 2-Ray[1].

Theorem 5.1 Every matroid of rank three satisfies (3/2)-Ray[2].

Proof. Let $\mathcal{M}$ be a matroid of rank three and let $\mathbf{y}>\mathbf{0}$ be positive weights on $E(\mathcal{M})$. Consider any four-element subset $S=\{a, b, c, d\}$ of $E$. There are three cases (up to permuting the elements of $S$ ):

(i) $\{a, b, c, d\}$ are collinear;

(ii) $\{a, b, c\}$ are collinear and $d$ is not on this line;

(iii) $\{a, b, c, d\}$ are in general position.

In case (i) it is easy to see that $\Psi_{3} M S=0$, so that the inequality $2 \Psi_{2} M S \geq 3 \Psi_{3} M S$ is trivially satisfied.

For cases (ii) and (iii) we consider the coefficient of each monomial in $\Psi_{2} M S$ and in $\Psi_{3} M S$ in turn.

First consider monomials of the form $y_{e}^{2}$ with $e \in E \backslash S$. The coefficient of $y_{e}^{2}$ in $\Psi_{2} M S$ is nonnegative. On the other hand, the coefficient of $y_{e}^{2}$ in $M_{b c d}^{a} M_{a}^{b c d}$ is zero, and 


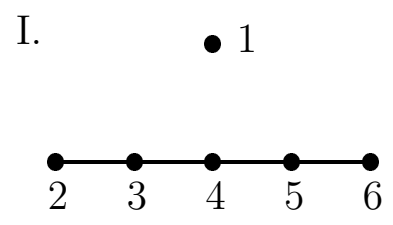

III.
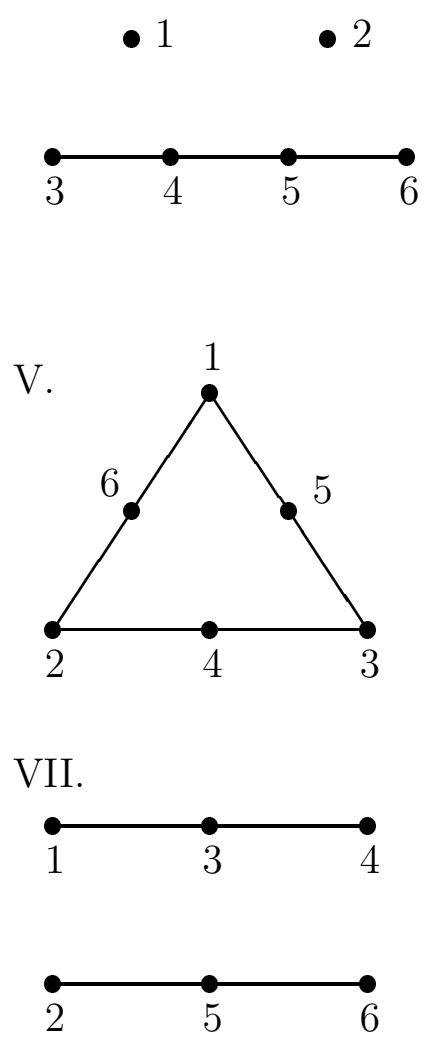
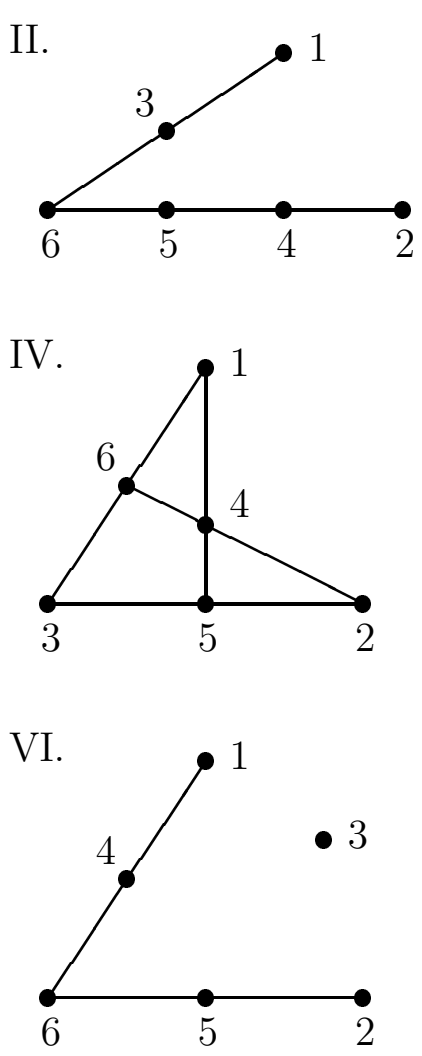

VIII.

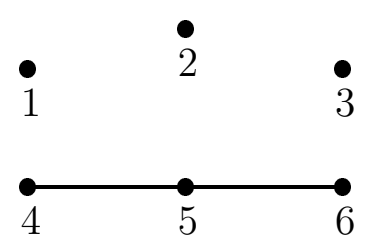

Figure 1: The six-element rank three matroids. 


\begin{tabular}{|r|r|r|c|}
\hline $\mathcal{N}\{a, b, c, d\}$ & $\Psi_{2}$ & $\Psi_{3}$ & $2 \Psi_{2}-3 \Psi_{3}$ \\
\hline $\mathrm{I}\{2,3,4,1\}$ & 0 & 0 & 0 \\
$\mathrm{II}\{1,3,6,2\}$ & 8 & 2 & 10 \\
$\mathrm{II}\{2,4,5,1\}$ & 6 & 3 & 3 \\
$\mathrm{II}\{2,4,6,1\}$ & 6 & 3 & 3 \\
$\mathrm{III}\{3,4,5,1\}$ & 6 & 3 & 3 \\
$\mathrm{IV}\{1,3,6,2\}$ & 8 & 2 & 10 \\
$\mathrm{~V}\{1,2,6,3\}$ & 8 & 3 & 7 \\
$\mathrm{~V}\{1,2,6,4\}$ & 10 & 3 & 11 \\
$\operatorname{VI}\{1,4,6,2\}$ & 10 & 3 & 11 \\
$\operatorname{VI}\{1,4,6,3\}$ & 12 & 3 & 18 \\
$\operatorname{VII}\{1,3,4,2\}$ & 12 & 3 & 15 \\
$\operatorname{VIII}\{4,5,6,1\}$ & 12 & 3 & 15 \\
\hline
\end{tabular}

Table 1: Monomials of shape $y_{e} y_{f}$ in case (ii).

similarly the coefficient of $y_{e}^{2}$ in $\Psi_{3} M S$ is zero. It follows that in both cases (i) and (ii), the coefficient of $y_{e}^{2}$ in $2 \Psi_{2} M S-3 \Psi_{3} M S$ is nonnegative.

Next consider monomials of the form $y_{e} y_{f}$ with $e \neq f$ in $E \backslash S$. The coefficiens of $y_{e} y_{f}$ in $\Psi_{2} M S$ and in $\Psi_{3} M S$ depend only on the restriction $\mathcal{N}:=\mathcal{M} \mid\{a, b, c, d, e, f\}$ and the position of $\{a, b, c, d\}$ within $\mathcal{N}$. Figure 1 lists all the rank three matroids on six elements, up to isomorphism. Table 1 lists the possible cases for $\mathcal{N}$ and $\{a, b, c, d\}$, up to isomorphism, in case (ii). Table 2 lists the possible cases for $\mathcal{N}$ and $\{a, b, c, d\}$, up to isomorphism, in case (iii). In each table the first column specifies $\mathcal{N}$ and $\{a, b, c, d\}$, the second column gives the coefficient of $y_{e} y_{f}$ in $\Psi_{2} M S$, and the third column gives the coefficient of $y_{e} y_{f}$ in $\Psi_{3} M S$. The fourth column computes the coefficient of the corresponding monomial of $2 \Psi_{2} M S-3 \Psi_{3} M S$. Only in case (iii) subcase IV $\{1,2,3,4\}$ do we obtain a negative coefficient.

Given a set $S=\{a, b, c, d\}$ in general position in $\mathcal{M}$, there are at most three points in $E \backslash S$ that can appear in a restriction of $\mathcal{M}$ containing $S$ in subcase $\operatorname{IV}\{1,2,3,4\}$; these are the points $e:=\overline{a b} \cap \overline{c d}, f:=\overline{a c} \cap \overline{b d}$, and $g:=\overline{a d} \cap \overline{b c}$. Notice that (when they exist) each of $y_{e}^{2}, y_{f}^{2}$ and $y_{g}^{2}$ occurs in $2 \Psi_{2} M S-3 \Psi_{3} M S$ with coefficient 8. If at most one of $e$, $f$, and $g$ exists in $E(\mathcal{M})$ then subcase $\operatorname{IV}\{1,2,3,4\}$ does not arise. If two exist - say $e$ and $f$ - then

$$
8 y_{e}^{2}+8 y_{f}^{2}-4 y_{e} y_{f}=6 y_{e}^{2}+6 y_{f}^{2}+2\left(y_{e}-y_{f}\right)^{2}
$$

can be used to absorb the term with negative coefficient into a square of a binomial. If all of $e, f$, and $g$ exist in $E(\mathcal{M})$ then

$$
\begin{aligned}
& 8 y_{e}^{2}+8 y_{f}^{2}+8 y_{g}^{2}-4 y_{e} y_{f}-4 y_{e} y_{g}-4 y_{f} y_{g} \\
= & 4 y_{e}^{2}+4 y_{f}^{2}+4 y_{g}^{2}+2\left(y_{e}-y_{f}\right)^{2}+2\left(y_{e}-y_{g}\right)^{2}+2\left(y_{f}-y_{g}\right)^{2}
\end{aligned}
$$

can be used to absorb the terms with negative coefficients into a sum of squares of binomials. 


\begin{tabular}{|r|r|r|c|}
\hline $\mathcal{N}\{a, b, c, d\}$ & $\Psi_{2}$ & $\Psi_{3}$ & $2 \Psi_{2}-3 \Psi_{3}$ \\
\hline $\mathrm{II}\{1,2,3,4\}$ & 8 & 2 & 10 \\
$\mathrm{III}\{1,2,3,4\}$ & 8 & 2 & 10 \\
$\mathrm{IV}\{1,2,3,4\}$ & 4 & 4 & -4 \\
$\mathrm{~V}\{1,2,4,5\}$ & 6 & 4 & 0 \\
$\mathrm{~V}\{1,4,5,6\}$ & 8 & 3 & 7 \\
$\mathrm{VI}\{1,2,4,5\}$ & 8 & 4 & 4 \\
$\mathrm{VI}\{1,2,3,4\}$ & 10 & 3 & 11 \\
$\mathrm{VI}\{1,2,3,6\}$ & 10 & 4 & 8 \\
$\mathrm{VII}\{1,2,3,5\}$ & 8 & 4 & 4 \\
$\mathrm{VIII}\{1,2,4,5\}$ & 10 & 4 & 8 \\
$\mathrm{IX}\{1,2,3,4\}$ & 12 & 4 & 12 \\
\hline
\end{tabular}

Table 2: Monomials of shape $y_{e} y_{f}$ in case (iii).

In each of cases (i), (ii), and (iii) we can thus write $2 \Psi_{2} M S-3 \Psi_{3} M S$ as a positive sum of monomials and squares of binomials in the variables $\mathbf{y}$. It follows that $\mathcal{M}$ satisfies the condition (3/2)-Ray[2].

Theorem 5.1, the result of [16], Lemma 4.2(c), and Corollary 4.5(b) immediately imply the following.

Theorem 5.2 Every matroid of rank three or corank three satisfies $\sqrt{\mathrm{BLC}}$.

As seen in [3], there are many matroids of rank three that are not HPP matroids - the Fano and Pappus matroids are two familiar examples. Theorem 5.2 begins to explore situations in which the hypothesis of Theorem 1.3 does not apply, but something close to its conclusion does hold. Unfortunately Theorem 5.2 implies nothing new about Mason's Conjecture, which is trivial in rank three.

\section{References}

[1] N. Balabanian and T.A. Bickart, "Electrical Network Theory," Wiley, New York, 1969.

[2] Y.-B. Choe, Rayleigh monotonicity of sixth-root of unity matroids, in preparation.

[3] Y.-B. Choe, J.G. Oxley, A.D. Sokal, and D.G. Wagner, Homogeneous polynomials with the half-plane property, Adv. in Applied Math. 32 (2004), 88-187.

[4] Y.-B. Choe and D.G. Wagner, Rayleigh matroids, http://www.arxiv.org/abs/math.C0/0307096, (to appear in Combin. Prob. Comput.). 
[5] A. Fettweis and S. Basu, New reults on stable multidimensional polynomials Part I: Continuous case, IEEE Trans. Circuits Systems 34 (1987), 1221-1232.

[6] T. Feder and M. Mihail, Balanced matroids, in "Proceedings of the 24th Annual ACM (STOC)", Victoria B.C., ACM Press, New York, 1992.

[7] C.D. Godsil, Real graph polynomials, in "Progress in graph theory (Waterloo, Ontario, 1982)", U.S.R. Murty and J.A. Bondy, eds., Academic Press, Toronto, 1984.

[8] G.H. Hardy, J.E. Littlewood, G. Pólya, "Inequalities" (Reprint of the 1952 edition), Cambridge U.P., Cambridge, 1988.

[9] G. Kirchhoff, Über die Auflösung der Gleichungen, auf welche man bei der Untersuchungen der linearen Vertheilung galvanischer Ströme geführt wird, Ann. Phys. Chem. 72 (1847), 497-508.

[10] R.D. Lyons, Determinantal probability measures, Publ. Math. Inst. Hautes Études Sci. 98 (2003), 167-212.

[11] J.H. Mason Matroids: unimodal conjectures and Motzkin's theorem, in "Combinatorics", D.J.A. Welsh and D.R. Woodall, eds., Oxford U.P., Oxford, 1972.

[12] R. Pemantle, Towards a theory of negative dependence, J. Math. Phys. 41 (2000), $1371-1390$.

[13] R. Schneider, On A.D. Aleksandrov's inequalities for mixed discriminants, J. Math. Mech. 15 (1966), 285-290.

[14] P.D. Seymour and D.J.A. Welsh, Combinatorial applications of an inequality from statistical mechanics, Math. Proc. Camb. Phil. Soc. 75 (1975), 495-495.

[15] R.P. Stanley, Two combinatorial applications of the Aleksandrov-Fenchel inequalities, J. Combin. Theory Ser. A 31 (1981), 56-65.

[16] D.G. Wagner, Rank three matroids are Rayleigh, http://www. arxiv.org/abs/math.C0/0403216, (to appear in Elec. J. Combin.).

[17] G. Whittle, On matroids representable over GF(3) and other fields, Trans. Amer. Math. Soc. 349 (1997), 579-603. 